\title{
ON THE CENTROID OF A HOMOGENEOUS WIRE
}

\author{
G. D. Chakerian and S. K. Stein
}

\section{INTRODUCTION}

Let $\mathrm{C}$ be a closed convex curve in the Euclidean plane $\mathrm{E}_{2}$. If $\mathrm{C}$ has continuous curvature, then the curvature centroid of $\mathrm{C}$ is defined as the center of mass of $\mathrm{C}$ considered as a wire whose density is equal to the curvature at each point. Hayashi [5] shows that at least four normals of $\mathrm{C}$ pass through its curvature centroid. Bose and Roy [2] and Tietze [6] prove that the area centroid of $\mathrm{C}$ has the same property (the area centroid is the center of mass of a disk of uniform density bounded by $\mathrm{C}$ ). In this paper, we prove that the perimeter centroid of $\mathrm{C}$ also has this property (Section 4). (The point $\left(\mathrm{x}_{0}, \mathrm{y}_{0}\right)$ is the perimeter centroid of $\mathrm{C}$ if

$$
\mathbf{L x}_{0}=\int_{\mathrm{C}} \mathrm{xds}, \quad \mathrm{Ly}_{0}=\int_{\mathrm{C}} \mathrm{yds},
$$

where $L$ is the length of $C$, and $s$ is arc length along C.) The proofs in [2] and [5] employ Fourier series and put restrictions on the smoothness of $\mathrm{C}$; however, even if $\mathrm{C}$ is assumed smooth, this technique fails to give the result for the perimeter centroid. Indeed, Bose and Roy [3] obtain by these methods only the weaker result that if $m$ is the number of points on $C$ where the radius of curvature is equal to three times the support function with respect to $\left(\mathrm{x}_{0}, \mathrm{y}_{0}\right)$, and $\mathrm{n}$ is the number of normals through $\left(x_{0}, y_{0}\right)$, then $m+n \geq 4$. The proof we give in Section 4 , like that of Tietze [6] in the case of the area centroid, is purely geometric and places no smoothness restrictions on $\mathrm{C}$.

The authors are indebted to the referee for several helpful suggestions.

\section{DEFINITIONS}

A support line of $\mathrm{C}$ is a line intersecting $\mathrm{C}$ so that the interior of $\mathrm{C}$ lies entirely on one side of the line.

A line or line segment $\ell$ containing a point $\mathrm{P}$ of $\mathrm{C}$ is a normal if and only if $\ell$ is orthogonal to some support line of $C$ through $P$.

Let $C_{1}$ be an arc lying in the upper half-plane and having its endpoints at $(-a, 0)$ and $(\mathrm{a}, 0)$ on the $\mathrm{x}$-axis. $\mathrm{C}_{1}$ is a convex arc if and only if, together with its chord from $(-a, 0)$ to $(a, 0)$, it forms a closed convex curve.

The moment of $\mathrm{C}_{1}$ about the $\mathrm{x}$-axis, denoted by $\mathrm{I}\left(\mathrm{C}_{1}\right)$, is given by

$$
\mathrm{I}\left(\mathrm{C}_{1}\right)=\int_{\mathrm{C}_{1}} \mathrm{yds}
$$

The integralgeometric definition of the area $A(S)$ of a surface $S$ in Euclidean space $E_{3}$ is as follows: let $d G$ be the usual integralgeometric density for the set of

Received November 26, 1963. 
lines $G$ in $E_{3}$ (see [1, p. 65] or [4, p. 88]). For each line $G$, let $n(G \cap S)$ be the number of points of intersection of $G$ with $S$. Then

$$
A(S)=\frac{1}{\pi} \int n(G \cap S) d G
$$

where the integration is over all lines G. It is proved in [1, p. 69], that this formula gives the "usual" surface area under suitable smoothness conditions on S. Moreover, in the case of convex surfaces the formula is consistent with the usual definition, without any smoothness restrictions (see [4, p. 95]). If $\mathrm{S}$ is the surface of revolution generated by revolving a convex arc $C$ about its chord, (3) is still consistent, as can be established by approximating $\mathrm{C}$ with inscribed polygons and considering the corresponding surfaces of revolution; however, in the proof of Lemma 1, below, we need (3) in this last case only when $C$ is a polygon.

\section{PRELIMINARY LEMMAS}

LEMMA 1. Let $\mathrm{C}_{1}$ and $\mathrm{C}_{2}$ be convex arcs in the upper half-plane, each having its endpoints at $(-\mathrm{a}, 0)$ and $(\mathrm{a}, 0)$. Suppose that $\mathrm{C}_{2}$ lies below $\mathrm{C}_{1}$ and also in the strip $\{(\mathrm{x}, \mathrm{y}):-\mathrm{a} \leq \mathrm{x} \leq \mathrm{a}\}$. Then, if $\mathrm{C}_{1} \neq \mathrm{C}_{2}$,

$$
\mathrm{I}\left(\mathrm{C}_{2}\right)<\mathrm{I}\left(\mathrm{C}_{1}\right)
$$

Proof. If $\mathrm{S}_{1}$ and $\mathrm{S}_{2}$ are the surfaces generated by revolving $\mathrm{C}_{1}$ and $\mathrm{C}_{2}$, respectively about the $x$-axis, then $S_{2}$ is a convex surface contained within $S_{1}$, and $S_{1} \neq S_{2}$. Moreover, the area of $S_{i}$ is given by

$$
A\left(S_{i}\right)=2 \pi I\left(C_{i}\right) \quad(i=1,2) .
$$

For any line $G, n\left(G \cap S_{2}\right) \leq n\left(G \cap S_{1}\right)$, and there exists a set of lines of positive measure intersecting $S_{1}$ but not intersecting $S_{2}$. Thus, the validity of formula (3) for $S=S_{1}$ would by (5) imply the required result (4). Instead of establishing (3) with $S=S_{1}$, we proceed as follows:

Let $P_{1}$ be a polygonal arc circumscribed about $C_{1}$ and having the same endpoints as $C_{1}$. Let $R_{1}$ be the surface generated by rotating $P_{1}$ about its chord. Then $R_{1}$ is the union of "conical segments" (cones and truncated cones), for each of which (3) is consistent. But the integral in (3) is additive, so that (3) is consistent for $R_{1}$. Hence we can apply the argument of the last paragraph, with $C_{1}$ replaced by $P_{1}$, to obtain the inequality

$$
\mathrm{I}\left(\mathrm{C}_{2}\right) \leq \mathrm{I}\left(\mathrm{P}_{1}\right)-\delta
$$

where $\delta$ is positive and independent of $\mathrm{P}_{1}$. (To establish the existence of $\delta$, one may consider the convex surface $S_{2}^{\prime}$ formed by taking the convex hull of $S_{2}$ and a point outside $S_{2}$ but inside $S_{1}$. Then $A\left(S_{2}\right)+2 \pi \delta=A\left(S_{2}^{\prime}\right) \leq A\left(R_{1}\right)$.) Now the continuity of $I(C)$, as a functional on convex arcs $C$, implies, by (6),

$$
\mathrm{I}\left(\mathrm{C}_{2}\right) \leq \mathrm{I}\left(\mathrm{C}_{1}\right)-\delta<\mathrm{I}\left(\mathrm{C}_{1}\right)
$$

We shall also use the following well-known, elementary lemma. 
LEMMA 2. Let $\mathrm{C}$ be a closed convex curve with the origin interior to $\mathrm{C}$, and let $\mathbf{r}=\mathrm{r}(\theta) \quad(0 \leq \theta \leq 2 \pi)$ be the polar equation of C. Suppose $\mathrm{r}(\theta)$ has a relative maximum or minimum. Then the corresponding radius is normal to $\mathrm{C}$.

\section{A FOUR-NORMAL THEOREM}

We now are ready to obtain the result described in the Introduction.

THEOREM. At least four normals to a closed convex curve pass through its perimeter centroid.

Proof. Assume that the perimeter centroid of $\mathrm{C}$ lies at the origin, so that

$$
\int_{C} y d s=0
$$

and let $\mathbf{r}=\mathbf{r}(\theta)(0 \leq \theta \leq 2 \pi)$ be the polar equation of $C$. If we can show that $r(\theta)$ has at least four relative maxima and minima, the theorem is proved, by Lemma 2. By periodicity, $r(\theta)$ must have an even number of extrema, so it suffices to show it is impossible that $r(\theta)$ has exactly one relative maximum and one relative minimum.

The continuity of $r(\theta)$ implies $r\left(\theta_{0}+\pi\right)=r\left(\theta_{0}\right)$ for some $\theta_{0}$. By performing a rotation, we may suppose $\theta_{0}=0$, so that $r(0)=r(\pi)=$ a. Suppose that $r(\theta)$ has exactly one maximum, assumed for $0<\theta<\pi$, and one minimum, necessarily assumed for $\pi<\theta<2 \pi$. Then $\mathrm{r}(\theta)>\mathrm{a}$ if $0<\theta<\pi$, and $\mathrm{r}(\theta)<\mathrm{a}$ if $\pi<\theta<2 \pi$. (Note that $\mathbf{r}(\theta) \neq \mathbf{a}$ for $0<\theta<\pi$, since otherwise at least two extrema would exist in $0<\theta<\pi$. The same holds for $\pi<\theta<2 \pi$.) It follows that the reflection across the $\mathrm{x}$-axis of the part of $\mathrm{C}$ in the lower half-plane is a convex arc $\mathrm{C}_{2}$ that lies below the arc $\mathrm{C}_{1}$ of $\mathrm{C}$ in the upper half-plane. Moreover, $\mathrm{C}_{2}$ lies in the disk $\left\{(x, y): x^{2}+y^{2} \leq a^{2}\right\}$, hence in the strip $\{(x, y):-a \leq x \leq a\}$. So by Lemma 1 , $\mathrm{I}\left(\mathrm{C}_{2}\right)<\mathrm{I}\left(\mathrm{C}_{1}\right)$. But then

$$
0<\mathrm{I}\left(\mathrm{C}_{1}\right)-\mathrm{I}\left(\mathrm{C}_{2}\right)=\int_{\mathrm{C}} \mathrm{yds}
$$

this contradicts (8) and completes the proof.

\section{SOME REMARKS}

Remark 1. The example of the ellipse shows that the number four in the theorem cannot be replaced by a larger number.

Remark 2. The converse of Lemma 2 is false, so one cannot conclude in general that the number of normals through a point is even. Indeed, we show how one can construct, for any integer $n \geq 5$, a convex curve $C$ with exactly $n$ normals through its perimeter centroid.

If $n$ is even ( $\mathrm{n} \geq 6$ ), a regular polygon of $n / 2$ sides does the trick. For $n$ odd, we proceed as follows. In the $(x, y)$-plane, let

$$
\mathrm{Q}=(0,1), \quad \mathrm{R}=(\mathrm{a}, 1), \quad \mathrm{S}=(\mathrm{b}, 0), \quad \mathbf{T}=(0,-1)
$$


where $0<a<b$. Let $C^{\prime}$ be the unit semicircle $\left\{(x, y): x^{2}+y^{2}=1, x \leq 0\right\}$. Let $C$ be formed by $C^{\prime}$ together with the segments QR, RS, and ST. Continuity considerations show that for the number a small, and a proper choice of $b$, the perimeter centroid $P$ lies on the line $x=a$ and in the upper half-plane. Exactly five normals of $C$ pass through $P$ (note that there is a normal at $R$ not corresponding to an extremum of the radial function). If one replaces $C^{\prime}$ by a rectangular arc $C^{\prime \prime}$ in the left half-plane with a pair of vertices at $Q$ and $T$, and a side parallel to the $y$-axis, such that $C^{\prime}$ and $C^{\prime \prime}$ have the same $x$ - and y-moments, then the perimeter centroid $P$ is unchanged. The new curve has seven normals passing through $P$. It is ciear that suitable variations of $C^{\prime}$ in this manner yield examples for any odd $\mathbf{n} \geq 5$.

Remark 3. Tietze's proof of the four-normal property for the area centroid is valid for any curve that is star-shaped with respect to its area centroid. On the other hand, the following example shows that, in general, even the simplest starshaped, nonconvex curves may fail to have four normals through the perimeter centroid.

Let $K$ be the circle $x^{2}+y^{2}=1$. Let $Q=(0,1 / 2)$. Let $R=\left(a, \sqrt{1-a^{2}}\right)$ $(0<a<1)$, and let $R^{\prime}$ be the reflection of $R$ across the $y$-axis. Let $C$ be the curve consisting of the larger arc $R R^{\prime}$ of $K$ together with the segments $R^{\prime} Q$ and $Q R$ (so that $C$ is a "notched" circle). If a is chosen small enough, the perimeter centroid $\mathbf{P}$ of $C$ lies on the positive half of the $y$-axis, $C$ is star-shaped with respect to $\mathrm{P}$, and exactly two normals of $\mathrm{C}$ pass through $\mathrm{P}$. By slightly rounding off the three corners of $\mathrm{C}$, one obtains an infinitely differentiable example.

\section{REFERENCES}

1. W. Blaschke, Vorlesungen über Integralgeometrie II, Chelsea, New York, 1949.

2. R. C. Bose and S. N. Roy, $A$ note on the area centroid of a closed convex oval, Bull. Calcutta Math. Soc. 27 (1935), 111-118.

3. - Some properties of the convex oval with reference to its perimeter centroid, Bull. Calcutta Math. Soc. 27 (1935), 79-86.

4. H. Hadwiger, Altes und Neues über konvexe Körper, Birkhäuser, Basel and Stuttgart, 1955.

5. T. Hayashi, Some geometrical applications of the Fourier series, Rend. Circ. Mat. Palermo 50 (1926); 96-102.

6. H. Tietze, Über die Anzahl der stabilen Ruhelagen eines Würfels, Elem. Math. 3 (1948), 97-100.

University of California, Davis 